\title{
The Gravitational Million-Body Problem
}

\author{
Douglas C. Heggie \\ University of Edinburgh, Department of Mathematics and Statistics, \\ King's Buildings, Edinburgh EH9 3JZ, UK
}

\begin{abstract}
We review what has been learned recently using $N$-body simulations about the evolution of globular clusters. While simulations of star clusters have become more realistic, and now include the evolution of single and binary stars, the prospect of reaching large enough $N$ is still a distant one. Nevertheless more restricted kinds of simulations have recently brought valuable progress for certain problems of current observational interest, including the origin and structure of tidal tails of globular clusters. In addition, such simulations have forced us to rethink some basic aspects of stellar dynamics, including, in particular, the process of escape. Finally we turn to faster, approximate methods for studying star cluster dynamics, where the role of $N$-body simulations is one of calibration.
\end{abstract}

\section{Introduction}

The title of this review unfortunately disguises the fact that it is about globular clusters, in the traditional sense. Actually, "a million" is somewhat on the large side in this context. Though the total number of stars in a globular cluster is not known, it may be estimated roughly by converting the luminosity (Harris 1996) to the mass, using the method of Mandushev, Staneva, \& Spasova (1991), and adopting a mean stellar mass of $0.34 M_{\odot}$, which corresponds approximately to a power-law mass function with slope -1 (Kroupa 2001) between turnoff and the hydrogen burning limit. The result is (Fig.1) that the median value is about $3 \times 10^{5}$, and roughly $10 \%$ of all clusters have more than one million stars. In this review we highlight some of the ways in which $N$-body simulations are being used in studying the dynamical theory of globular star clusters.

\section{Comprehensive Calculations ${ }^{1}$}

No simulation of a globular cluster will ever be complete, but rapid strides are being made to extend the traditional techniques of stellar dynamics so as to include stellar evolution. As a result there is a growing body of simulations with the following typical characteristics: (i) 1 star is represented by 1 particle

\footnotetext{
${ }^{1}$ At Tokyo several people expressed some distaste for the phrase "kitchen-sink calculations",
} which I used in my talk. 


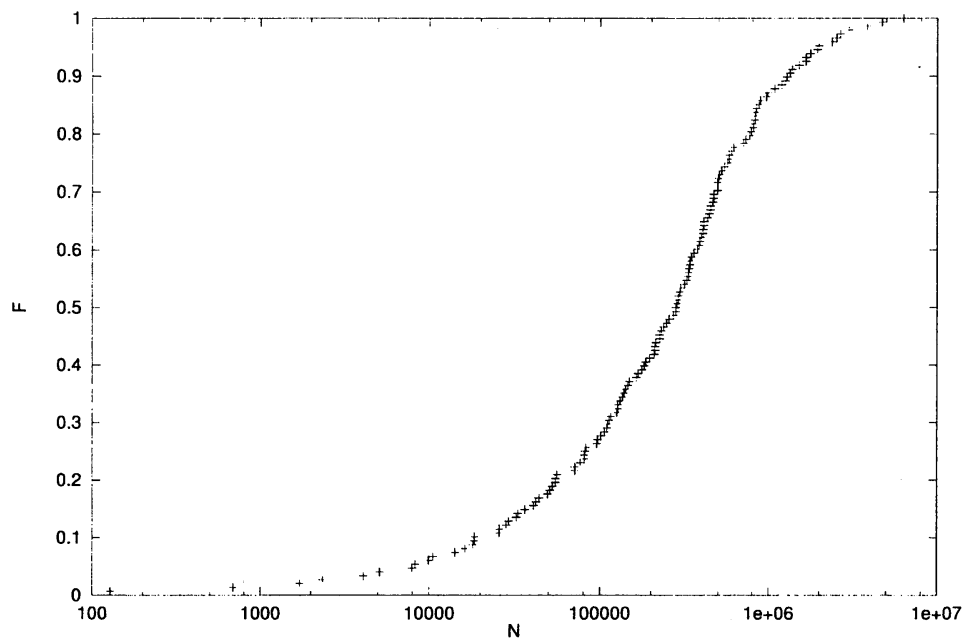

Figure 1. Cumulative distribution of approximate number of stars in the clusters of the galactic globular cluster system, derived as in the text.

("star-by-star calculation"); (ii) forces include star-star gravity, a galactic tide (for a cluster on either a circular or elliptic galactic orbit), and disk shocking; (iii) treatment of stellar collisions; (iv) inclusion of stellar properties (luminosity, colour, metallicity) and stellar evolution; and (v) inclusion of (primordial) binaries and binary evolution.

There is a strong synergy between the development of such codes and the development of special-purpose hardware (see the papers by Aarseth, Makino and the "starlab" group in this volume.) Table 1 lists some recent comprehensive simulations, where $f_{b i n}$ is the initial fraction of binaries. The scientific value of such work is well illustrated by Hurley et al (2001), who quantified the role of dynamical influences on the population of blue stragglers in an old open cluster. Such a result has been a long standing goal of this programme of research.

Table 1. Some comprehensive calculations

\begin{tabular}{llrr}
\hline Author & Object & $N(0)$ & $f_{\text {bin }}$ \\
\hline Hurley et al (2001) & M67 & 15000 & $50 \%$ \\
Portegies Zwart et al (2001a) & Pleiades, etc & 3000 & $50 \%$ \\
Portegies Zwart et al (2001b) & Arches, etc & 12000 & $0 \%$ \\
Kroupa et al (2001)* & "Pleiades" & 10000 & $100 \%$ \\
\hline \hline
\end{tabular}

*No Roche Lobe mass transfer, etc

Despite such successes, it is disappointing that not one of these is a simulation of a globular cluster, in the traditional sense of the term. Even with GRAPE-4 it was possible to study a model with $N=32768$ well past core col- 
lapse (Makino 1996), which includes at least the smallest $10 \%$ of the existing galactic globular clusters.

There are two reasons why simulation of any globular cluster is much harder than is suggested by the particle number alone. One is the requirement for a substantial population of primordial binaries. Though it has been argued that the computational load associated with binaries becomes negligible when $N$ is sufficiently large (Makino \& Hut 1990), this asymptotic limit is not yet in sight. The second reason is that the present particle number in a globular cluster may be a poor guide to what is required. Hurley et al (2001) estimated that even M67 had 7 times as many stars at birth as at present, and were therefore unable to simulate its early evolution. The present day mass function of the globular cluster NGC 6712 can be understood if it has lost about $99 \%$ of its original mass (Takahashi \& Portegies Zwart 2000), and since its current mass is estimated to be of order $10^{5} M_{\odot}$ (Pryor \& Meylan 1993), its likely original particle number puts it far beyond reach.

One conclusion from this discussion is that, if a comprehensive simulation of a globular cluster is carried out within the next few years, it will tell us only about the evolution of the few clusters (if there are any) which are of low mass now and always have been. Study of the dynamical evolution of globular clusters cannot wait until such simulations can cope with all clusters.

One of the aims of comprehensive simulations, as we have seen, is the study of the interaction between stellar and dynamical evolution, and the effects of collisions. While the dynamics of point masses can be computed with precision, the effect of collisions is very uncertain. As Sills et al (2001) make clear, the effects of rotation on a merger remnant are dramatic. Since this is likely to be the largest source of uncertainty in studying the effects of collisions in globular clusters, it may be perfectly acceptable to adopt a model in which the point-mass dynamics is treated approximately, such as a Monte Carlo model (Sec.5). These are much faster than direct $N$-body methods, and can reach much larger "particle" numbers, ensuring that rare (but observable) species are not neglected.

\section{Restricted Simulations}

Considerable progress has been made in recent years in understanding important aspects of the dynamics of globular clusters without attempting a comprehensive simulation. The example we shall concentrate on here is the simulation of tidal tails. It is a theoretical problem that has been stimulated by observations (Grillmair et al 1995, 1996; Kharchenko, Scholz, \& Lehmann 1997; Leon, Meylan, \& Combes 2000; Testa et al 2000; Odenkirchen et al 2001). For this purpose one need include only (i) point mass dynamics, (ii) relaxation, (iii) the galactic tide, and (iv) the galactic orbit. Binaries and stellar evolution can be ignored. This is a pure stellar dynamics problem, and several sets of simulations have been carried out: Moore (1996); Combes, Leon \& Meylan (1999), Murali \& Dubinski (1999), Johnston, Sigurdsson \& Hernquist (1999), Dehnen (pers. comm., and Fig.2), and others. The list would be much longer if one added comparable work on satellite galaxies. 


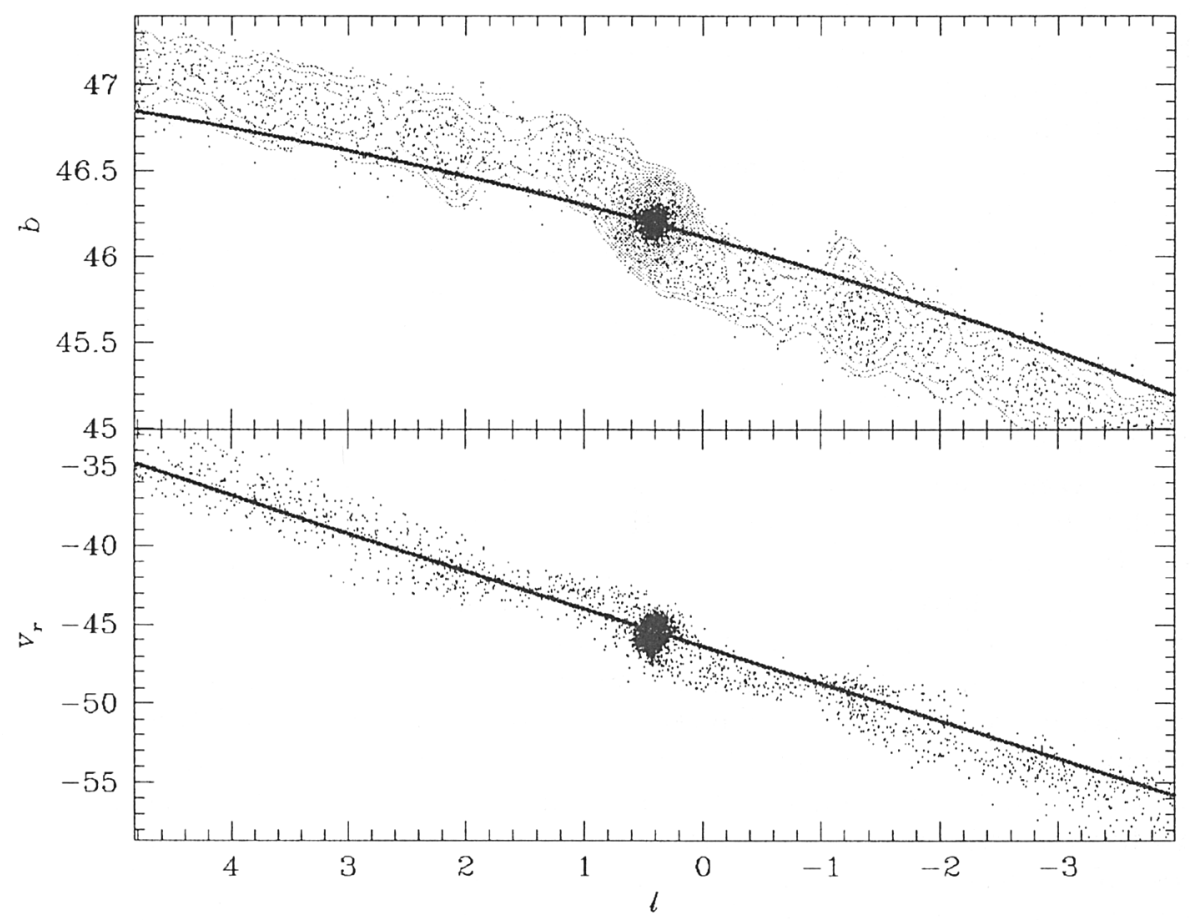

Figure 2. Simulation of Pal 5 (Dehnen, pers. comm., reproduced by kind permission of the author). The orbit chosen is consistent with the present position, radial velocity and proper motion, and used the galactic potential of model 3 in Dehnen \& Binney (1998). The initial condition is a King model with $N=10^{4}$. Upper panel: position on the sky $(l, b)$; lower panel: radial velocity against longitude. Note the clumps in the tail in the upper panel; they resemble those in Pal 5 itself (Odenkirchen et al 2001, where they are attributed to recent disk shocks). 
What is not always clear from simulations is the mechanism by which stars escape from a cluster into the tidal tail. The most obvious possibilities are timedependent tides (disk and bulge shocking) and relaxation. But such simulations usually cover only one or two Gyr of evolution, and it is possible that the choice of initial conditions plays a role, just as in the case of circular orbits (Fukushige \& Heggie 2000). The other hard thing to get right is relaxation, though the simulations of Combes et al (1999) show that this can be done.

The role of these various mechanisms can be considerably clarified by a judicious choice of initial conditions and parameters. Baumgardt (this volume) begins at apogalacticon, with a cluster whose radius corresponds to the Roche radius at perigalacticon. The rationale for this is not the assumption that, over the course of many galactic orbits, the radius of a cluster is set by its Roche radius at perigalacticon; such an assumption would be in contradiction with observational evidence (Ninkovic 1985, Allen \& Martos 1988, Meziane \& Colin 1996; Brosche, Odenkirchen \& Geffert 1999). Rather, if the initial radius of the cluster is either much greater or much smaller than the perigalactic tidal radius, then it is clear that initial conditions have much to do with the subsequent mass loss.

Baumgardt's finding from his $N$-body simulations is that, if one estimates the lifetime on the basis of mass lost in the first few orbits, as is often done, one finds that the lifetime increases with $N$ roughly as the relaxation time, until sufficiently large $N$ is reached, and then the lifetime is approximately independent of $N$. This is what would be expected if relaxation dominates for small $N$ and tidal heating dominates for large $N$. If, however, one waits for as many galactic orbits as are required to lose a certain fraction (such as $25 \%$ ) of the mass, one finds that the mass loss is dominated by relaxation, for all $N$ up to the largest values he was able to study. Though this $N$ is still much smaller than the number of stars in the larger globular clusters, Baumgardt's $N$-body models used particles of equal mass, and their relaxation time actually corresponds to the relaxation time of a much larger cluster with unequal masses.

One draws two conclusions from this study. One is that the initial conditions matter; it is only after the lapse of a sufficient number of galactic orbits that the mass loss rate reaches its asymptotic size. Second, the escape rate is dominated by relaxation, and not by bulge shocking, even on a very eccentric galactic orbit. It remains to be seen, however, whether disk shocking is ever important.

\section{New Stellar Dynamics}

While it is clear that there are quantitative questions which can only be answered from $N$-body simulations, has anything qualitatively new come out of them? Indeed, yes, but the list is not a long one. Most recently, we have learned that the lifetime of clusters does not scale with $N$ in the expected manner, and the present section summarises this discovery.

The aim of a collaborative experiment carried out in 1997 (Heggie et al 1998; Heggie, this vol.) was to compare results of various kinds of codes (and not only $N$-body codes) in studying the dynamical evolution of a specific object. Since the number of particles was of order 250000 , it was necessary to scale $N$-body simulations by $N$. When this was done by the relaxation time, it was found 
that the lifetime of the system depended on $N$, though its internal evolution (e.g. time to core collapse) was fairly insensitive to $N$.

Fukushige suggested (pers. comm.) that the problem lay in the time taken for stars to escape, once they had enough energy to do so. If, however, it was assumed that the escape time scale is proportional to the crossing time, $t_{c r}$, then it seemed hard to understand how the problem persisted for the large values of $N$ (up to $64 \mathrm{~K}$ ) for which results were available. The lifetime of a cluster should vary nearly with the relaxation time, except for $N$ up to a few hundred, as in the escape theory of King (1959). Fukushige \& Heggie (2000), however, found that the time taken to escape is a sensitive function of the energy, $E$, of a star: $t_{e s c} \propto\left(E-E_{e s c}\right)^{-2}$, where $E_{e s c}$ is the energy needed to escape (i.e. the potential at the Lagrange point, where the potential well of the cluster opens out into the rest of the galaxy).

The next question was to find the appropriate energy to use in this formula. The answer is provided in Baumgardt (2001a). (His argument is more detailed than the following, but leads to the same scalings.) Unescaped stars with energies just above $E_{\text {esc }}$ are relaxing during the time they take to escape, and so their excess energy is $E-E_{e s c} \propto\left(t_{e s c} / t_{r l x}\right)^{1 / 2}$, since relaxation is a diffusion process. (Here, $t_{r l x}$ is the relaxation time.) By solving these two relations we find the following conclusions: (i) $t_{e s c} \sim\left(t_{c r} t_{r l x}\right)^{1 / 2}$, much longer than the crossing time, and quite different from previous expectations; this helps to explain why the effects of the escape time persist for much larger $N$ than expected; (ii) $E-E_{e s c} \propto t_{r l x}^{-1 / 4}$; this is also the fraction of stars inside the cluster with energy above $E_{e s c}$; this fraction is still a few percent, even for systems of the size of a typical globular cluster; and (iii) from the previous two conclusions, the time to lose some fraction (say, half) of the mass of the cluster is $t_{M} \propto t_{r l x}^{3 / 4}$.

The last is the most far-reaching conclusion. Though the theory applies only for circular galactic orbits, Baumgardt has shown empirically (these proceedings) that the same conclusion appears to hold for clusters on elliptic galactic orbits. And yet Vesperini \& Heggie (1997) studied the evolution of the mass function of clusters assuming that $t_{M} \propto t_{r l x}$, which is now known to be wrong. Indeed a great deal of recent modelling of globular cluster systems (e.g. Capuzzo-Dolcetta \& Tesseri 1997, Gnedin \& Ostriker 1997, Murali \& Weinberg 1997, and other work of authors already quoted) may require revision. In effect, the new scaling of $t_{M}$ means that the lifetime of a globular cluster through dynamical processes is smaller (for large $N$ ) than one would have thought. Baumgardt (2001b) shows that one consequence of this is that the initial conditions of the cluster system are more completely erased.

\section{Calibration of Faster Methods}

\subsection{Faster methods for star cluster dynamics}

Besides $N$-body simulations, there are several other methods for studying the dynamical evolution of globular clusters (Fig.3). There are scaling methods, in which the cluster is characterised by nothing more than its mass and length scale (for example), Fokker-Planck methods, gas methods, and various hybrids. Even tree methods may function well for some important problems (McMillan \& 


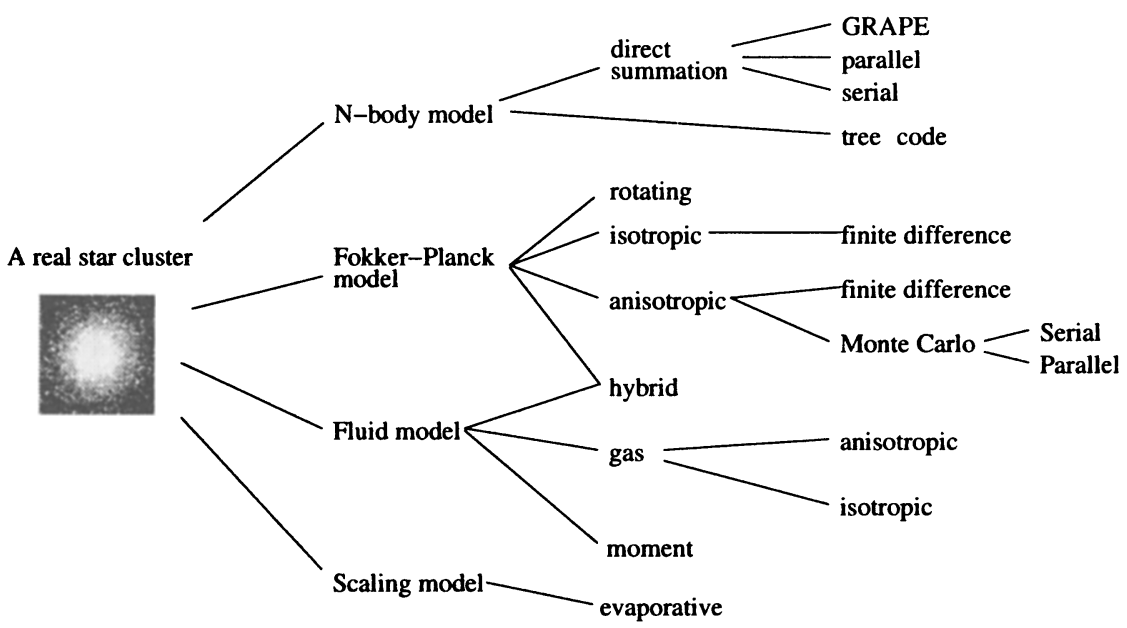

Figure 3. Models of the dynamics of dense stellar systems. We do not include all hybrids, or methods used in other areas of stellar dynamics.

Aarseth 1993, Arabadjis \& Richstone 1998, Miocchi \& Capuzzo-Dolcetta 2001). All these methods are faster and involve more approximations, and $N$-body simulations are required in order to calibrate them.

We have already seen (Sec.2) that such methods may be quite appropriate for problems where the accuracy of the stellar dynamics is not the main uncertainty. Fast methods are also appropriate for the following kinds of problems:

1. Evolution of cluster systems: For references see Sec.4. For this purpose it must be possible to study the dynamical evolution of many globular cluster models with the full range of realistic $N$. At present this is possible only with fast approximate methods.

2. Modelling individual objects: Fokker-Planck and (occasionally) fluid methods have been used to construct evolutionary models for comparison with observations of specific clusters, including M3 (Angeletti, Dolcetta \& Giannone 1980), M71 (Drukier, Fahlman \& Richer 1992), NGC 6624 (Grabhorn et al 1992), NGC 6397 (Drukier 1993, 1995), M15 (Grabhorn et al 1992, Phinney 1993, Dull et al 1997) and M30 (Howell, Guhathakurta \& Tan 2000). This requires extensive trial and error, with variations of the initial conditions of the model, and fast methods are essential. One motivation for such work has been the need to select suitable observational fields in clusters such that the local mass function requires least correction to the global mass function.

3. Predicting initial conditions: This is the theorists' counterpart of the previous point. If one wants to construct an $N$-body model of a given object, considerable trial and error is needed in order to find appropriate initial conditions. Faster methods should be able to give a rapid but approximate answer, which can then be refined with a minimal number of $N$-body simulations. 


\subsection{Recent examples}

Scaling models As part of a study of the dynamical evolution of the mass function of a star cluster, Vesperini \& Heggie (1997) gave a simple formula for the evolution of the mass, $M(t)$, i.e. $M(t)=M(0)-\Delta M_{\text {s.e. }}-0.828 M(0) t / F_{C W}$, where $\Delta M_{\text {s.e. }}$ is the contribution from stellar evolution (which also depends on the mass function), and $F_{C W}$ is the "family" parameter of Chernoff \& Weinberg (1990). For a given mass function, it is proportional to the relaxation time for average conditions inside the tidal radius, and is defined by $F_{C W}=\left(M / M_{\odot}\right)\left(R_{g} / \mathrm{kpc}\right)\left(220 \mathrm{kms}^{-1} / v_{g}\right) \gamma^{3 / 2} / \ln N$, where $M$ is the (original) mass of the cluster, moving on an orbit of radius $R_{g}$ at speed $v_{g} . \gamma$ depends on the distribution of mass in the galaxy, is unity for a point-mass galaxy, and $(3 / 2)^{1 / 3}$ for an isothermal potential.

The above formula for $M(t)$ has been used by Vesperini $(1998,2000,2001)$ in studies of the evolution of cluster systems. Its relevance to the present review is that it was based on the simplest scaling considerations, but the numerical coefficient was obtained by examination of $N$-body simulations. In other words, $N$-body results were used to calibrate a simpler theoretical model.

We now briefly consider the lifetime of a cluster. In the absence of stellar evolution, the above formula gives a cluster lifetime $t_{V H}=F_{C W} / 0.828$ in Myr. The coefficient depends weakly on the structure of the initial model. A similar formula for $M(t)$ has been given by Portegies Zwart et al (2001b), which leads to a lifetime $t_{P Z M M H}=0.29 t_{r x t}$. Here $t_{r x t}$ is another measure of the relaxation time within the tidal radius, defined by $t_{r x t}=0.138 M^{1 / 2} r_{t}^{3 / 2} /\left(\bar{m} G^{1 / 2} \log _{10} \Lambda\right)$ (cf. Spitzer 1987, eq.(2-63), except that Spitzer uses the natural logarithm in the Coulomb factor $\log \Lambda$ ). In this formula, $\bar{m}$ is the mean stellar mass.

For the galactic mass law used by Portegies Zwart et al, $\gamma=(3 / 1.8)^{1 / 3}$, and so it is easy to show, if we equate the two forms of the Coulomb logarithm, that the ratio of the lifetimes is $t_{V H} / t_{P Z M M H} \simeq 0.34 M_{\odot} / \bar{m}$. The dependence on $\bar{m}$ arises because $F_{C W} / t_{r x t}$ also depends on $\bar{m}$. If it is assumed that the lifetime is proportional to $t_{r x t}$ (independent of the mass function, cf. de la Fuente Marcos $1995)$, then it is necessary to modify $t_{V H}$ to $t_{V H}=\left(F_{C W} / 0.828\right)\left(\bar{m}_{V H} / \bar{m}\right)$, where $\bar{m}_{V H}$ is the value in the simulations by Vesperini \& Heggie (1997). In fact for most of their work, which used a mass function $f(m) \propto m^{-2.5}$ for $0.1 M_{\odot}<m<15 M_{\odot}$ we have $\bar{m}_{V H} \simeq 0.28 M_{\odot}$. The effect of this change (which is also necessary in the formula for $M(t)$ ) is to bring the two independent estimates of cluster lifetime into fair agreement. It must be borne in mind, however, that the assumption that the lifetime is proportional to the relaxation time is incorrect (Sec.4), and that $\bar{m}$ is time-dependent.

Despite the fact that lifetime (in the absence of stellar evolution) does not depend much on the initial structure (if the cluster initially fills its Roche lobe), it would be useful to have an equally simple way of estimating the time-dependence of the concentration parameter.

Fokker-Planck models The comparison between $N$-body and Fokker-Planck models (computed with finite differences) has a considerable history, but recent work appears to have brought the two methods into reasonable agreement, with one caveat, discussed below. The central issue is the escape criterion, as shown by Takahashi \& Portegies Zwart $(1998,2000)$. These papers show how a free 
parameter in the Fokker-Planck specification can be determined by calibration using the results of $N$-body models. Incidentally, the second of these is also an extensive survey of globular cluster models, which essentially supersedes the comparable extensive Fokker-Planck work of Chernoff \& Weinberg (1990).

As Takahashi \& Portegies Zwart themselves point out, the caveat in this is that the $N$-body models used in this survey adopt a tidal cutoff. Since it is known that the lifetime varies with $N$ in a different way when a tidal field is used (Sec.4), it remains to be seen how well the agreement between Fokker-Planck and $N$-body models survives in the more realistic case.

Monte Carlo models Though these can be regarded also as Fokker-Planck models, they offer some advantages over finite-difference models, and have received increasing attention in recent years. While part of this effort involves calibration with $N$-body models, the real purpose of this little subsection is to say something about the present state of play.

The largest models to date are those of Freitag \& Benz (2001), who present results of Monte Carlo simulations with up to $2 \times 10^{6}$ shells. At such levels it becomes possible to represent many globular clusters with one shell per star. These models, which are motivated by problems of galactic nuclei, do not include binaries, but have been extended to include stellar collisions (Fig.4). For models including the effects of dynamically formed binaries the record is held, at time of writing, by Giersz (this volume). Reaching well past core collapse with $N=10^{6}$ shells, as he does, is a notable landmark. Primordial binaries are excluded from these models, but a $10 \%$ population of primordial binaries are present in computations with up to $3 \times 10^{5}$ shells by Rasio's group (Rasio, Fregeau \& Joshi, 2001; Fig.5). Soon they will compute few-body interactions "on the fly", instead of using cross sections. All these developments give great promise for the Monte Carlo method in the coming years.

\section{Conclusions}

This rather selective review has concentrated on what $N$-body models are telling us about the dynamics of globular star clusters. Comprehensive simulations, which include details of evolution of single and binary stars, are at an exciting stage, but are still limited to rich open clusters. It seems unlikely that comprehensive simulations of globular clusters will be possible in the near future. Much progress can be made on some interesting kinds of dynamical problems about globular clusters (e.g. tidal tails) using more restricted kinds of simulation, which are perfectly feasible now. One of the dynamical lessons that has been learned in recent years is that it is vital to implement a correct treatment of tidal effects, even in the case of a steady tide (i.e. a circular galactic orbit). Monte Carlo methods now have the dynamical aspects of globular clusters within reach. When a detailed treatment of stellar evolution has been incorporated (at the level which has already been successful in the best $N$-body codes), these codes may well prove optimal for the comprehensive study of globular star clusters, until the time when real $N$-body models are fast enough.

Acknowledgments. I thank the organisers of the meeting for their support, and I am grateful for comments from H. Baumgardt, S.P.F. Portegies 
(a)

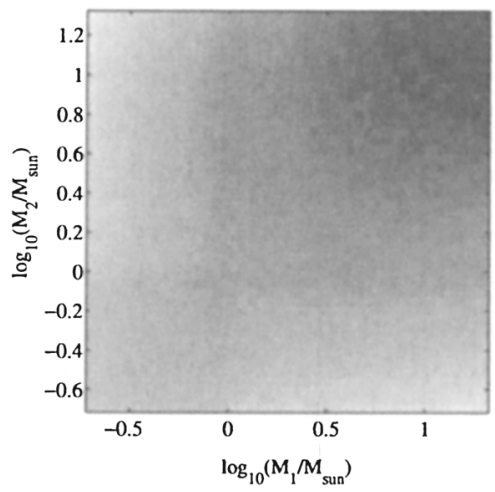

(b)

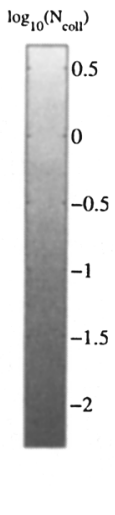

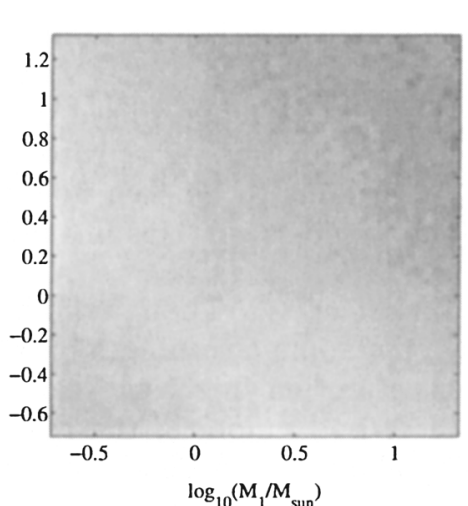

Figure 4. Comparison of the collision rate between stars of mass $M_{1}$ and $M_{2}$ in a static Plummer model (left) and in a Monte Carlo simulation (right) with 512000 shells, but without stellar evolution (from Freitag 2000, with kind permission). The collision rate among more massive stars is greatly enhanced by mass segregation.

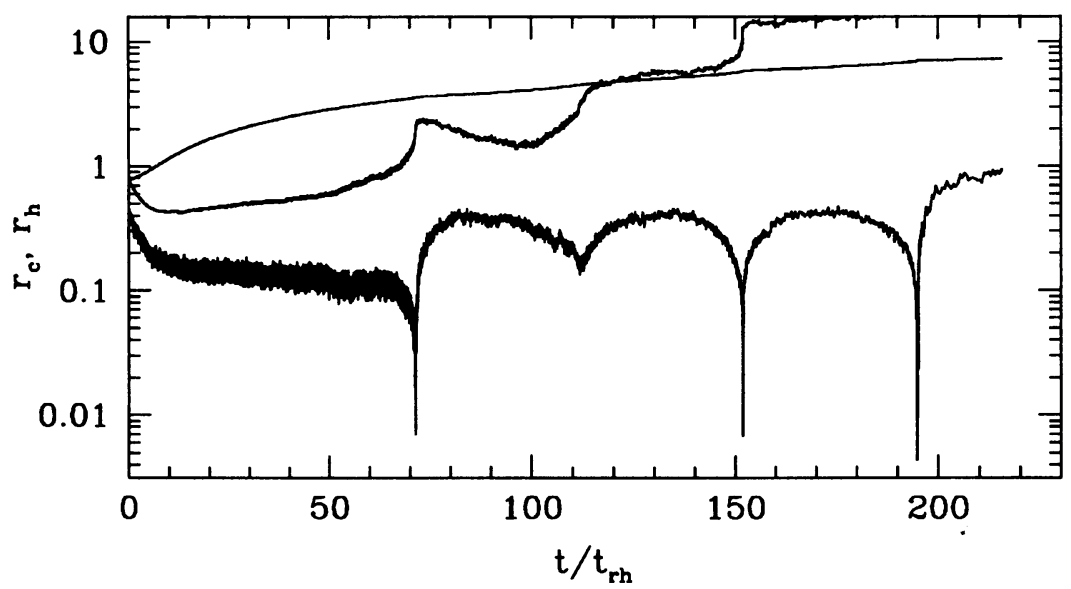

Figure 5. Simulation of an isolated system with $N=3 \times 10^{5}$ initially, including $10 \%$ primordial binaries (F. Rasio, by kind permission). Radii plotted against time are, from the bottom: core radius, half-mass radius of binaries, half-mass radius of all stars. Core collapse is avoided for about 70 initial half-mass relaxation times, when gravothermal oscillations begin. 
Zwart and E. Vesperini on scientific matters. I also thank W. Dehnen, M. Giersz, M. Freitag and F. Rasio for the provision of unpublished results. The picture of M15 in Fig.3 was obtained using NASA's SkyView facility ${ }^{2}$ located at NASA Goddard Space Flight Center.

\section{References}

Allen, C. \& Martos, M. A. 1988, Revista Mexicana de Astronomia y Astrofisica, 16,25

Angeletti, L., Dolcetta, R., \& Giannone, P. 1980, Ap\&SS, 69, 45

Arabadjis, J.S. \& Richstone D.O. 1998, astro-ph/9810192

Baumgardt, H. 2001a, MNRAS, 325, 1323

Baumgardt, H. 2001b, in 'Modes of Star Formation', eds. E. Grebel \& W. Brandner, ASP Conf. Ser (ASP, San Francisco), in press

Brosche, P., Odenkirchen, M., \& Geffert, M. 1999, New Astronomy, 4, 133

Capuzzo-Dolcetta, R. \& Tesseri, A. 1997, MNRAS, 292, 808

Chernoff, D. F. \& Weinberg, M. D. 1990, ApJ, 351, 121

Combes, F. , Leon, S. , \& Meylan, G. 1999, A\&A, 352, 149

Dehnen, W. \& Binney, J. 1998, MNRAS, 294, 429

de la Fuente Marcos, R. 1995, A\&A, 301, 407

Drukier, G. A. 1993, MNRAS, 265, 773

Drukier, G. A. 1995, ApJS, 100, 347

Drukier, G. A., Fahlman, G. G., \& Richer, H. B. 1992, ApJ, 386, 106

Dull, J. D., Cohn, H. N., Lugger, P. M., Murphy, B. W., Seitzer, P. O., Callanan, P. J., Rutten, R. G. M., \& Charles, P. A. 1997, ApJ, 481, 267

Freitag, M. 2000, PhD thesis, Université de Genève; http://obswww.unige.ch/ freitag/thesis/

Freitag, M., \& Benz, W. 2001, A\&A, 375, 711

Fukushige, T. \& Heggie, D. C. 2000, MNRAS, 318, 753

Gnedin, O. Y. \& Ostriker, J. P. 1997, ApJ, 474, 223

Grabhorn, R.P., Cohn, H.N., Lugger, P.M., \& Murphy, B.W. 1992, ApJ, 392, 86

Grillmair, C. J., Freeman, K. C., Irwin, M., \& Quinn, P. J. 1995, AJ, 109, 2553

Grillmair, C. J., Ajhar, E. A., Faber, S. M., Baum, W. A., Holtzman, J. A., Lauer, T. R., Lynds, C. R., \& O'Neil, E. J. 1996, AJ, 111, 2293

Harris, W.E. 1996, AJ, 112, 1487

Heggie, D. C., Giersz, M., Spurzem, R., \& Takahashi, K. 1998, Highlights in Astronomy, 11, 591

Howell, J. H., Guhathakurta, P., \& Tan, A. 2000, AJ, 119, 1259

Hurley, J.R., Tout, C.A., Aarseth, S.J., Pols, O.R. 2001, MNRAS, 323, 630

${ }^{2}$ http://skyview.gsfc.nasa.gov 
Johnston, K. V., Sigurdsson, S., \& Hernquist, L. 1999, MNRAS, 302, 771

Kharchenko, N., Scholz, R.-D., \& Lehmann, I. 1997, A\&AS, 121, 439

King, I. 1959, AJ, 64, 351

Kroupa, P. 2001, in S. Deiters, R. Spurzem, et al., eds, STAR2000: Dynamics of Star Clusters and the Milky Way, ASP Conf. Ser. (ASP, San Francisco), in press; astro-ph/0011328

Kroupa, P., Aarseth, S., Hurley, J. 2001, MNRAS, 321, 699

Leon, S., Meylan, G., \& Combes, F. 2000, A\&A, 359, 907

Makino, J. 1996, ApJ, 471, 796

Makino, J., Hut, P. 1990, ApJ, 365, 208

Mandushev, G., Staneva, A., \& Spasova, N. 1991, A\&A, 252, 94

McMillan, S. L. W. \& Aarseth, S. J. 1993, ApJ, 414, 200

Meziane, K. \& Colin, J. 1996, A\&A, 306, 747

Miocchi, P. \& Capuzzo-Dolcetta, R. 2001, A\&A, submitted; astro-ph/0104152

Moore, B. 1996, ApJ, 461, L13

Murali, C. \& Dubinski, J. 1999, AJ, 118, 911

Murali, C. \& Weinberg, M. D. 1997, MNRAS, 291, 717

Ninkovic, S. 1985, AN, 306, 237

Odenkirchen, M. et al. 2001, ApJ, 548, L165

Phinney, E.S. 1993, in S.G. Djorgovski and G. Meylan, eds, Structure and Dynamics of Globular Clusters, ASP Conf. Ser. 50 (ASP, San Francisco)

Portegies Zwart, S.F., McMillan, S.L.W., Hut, P., Makino, J. 2001a, MNRAS, 321,199

Portegies Zwart, S.F., Makino, J., McMillan, S.L.W., Hut, P. 2001b, ApJ, 546L, 101

Pryor, C. \& Meylan, G. 1993, in S.G. Djorgovski and G. Meylan, eds, Structure and Dynamics of Globular Clusters, ASP Conf. Ser. 50 (ASP, San Francisco)

Rasio, F.A., Fregeau, J.M., Joshi, K.J. 2001, in 'The Influence of Binaries on Stellar Population Studies', ed. D. Vanbeveren (Kluwer, Dordrecht), in press; astro-ph/0103001

Sills, A., Faber, J.A., Lombardi, J.C., Rasio, F.A., \& Warren, A.R. 2001, ApJ, 548,323

Spitzer, L., Jr., 1987, Dynamical Evolution of Globular Clusters (Princeton, Princeton University Press)

Takahashi, K. \& Portegies Zwart, S.F. 1998, ApJ, 503, L49

Takahashi, K. \& Portegies Zwart, S.F. 2000, ApJ, 535, 759

Testa, V., Zaggia, S. R., Andreon, S., Longo, G., Scaramella, R., Djorgovski, S. G., \& de Carvalho, R. 2000, A\&A, 356, 127

Vesperini, E. 1998, MNRAS, 299, 1019

Vesperini, E. 2000, MNRAS, 318, 841

Vesperini, E. 2001, MNRAS, 322, 247

Vesperini, E. \& Heggie, D. C. 1997, MNRAS, 289, 898 